\title{
eMED-DNA: An in silico operating system for clinical medical data storage within the human genome
}

Md. Jakaria ${ }^{1}$, Kowshika Sarker ${ }^{1}$, Mostofa Rafid Uddin ${ }^{1}$, Md. Mohaiminul Islam ${ }^{1}$, Trisha Das ${ }^{1}$, Rameen Shakur ${ }^{2,3 *}$, Md. Shamsuzzoha Bayzid ${ }^{1, *}$

${ }^{1}$ Bangladesh University of Engineering and Technology. Computer Science and Engineering, Dhaka, Bangladesh -1205.

${ }^{2}$ The Koch institute for integrative cancer research at MIT. Massachusetts institute of technology 77 Massachusetts Ave, Cambridge, MA 02139, United States

${ }^{3}$ Wellcome Sanger Institute. Wellcome Genome Campus. Hinxton. Cambridge CB10 1SA, UK

* Corresponding Authors.

The first five authors contributed equally and share the first-authorship of this paper.

Keywords: Precision medicine, DNA storage, File management system, Genomic medicine, Translational technologies, Genome sequence, Non-coding regions, Next generation sequencing, Health Informatics. 


\begin{abstract}
The propitious developments in molecular biology and next generation sequencing have enabled the possibility for DNA storage technologies. However, the full application and power of our genomic revolution have not been fully utilized in clinical medicine given a lack of transition from research to real world clinical practice. This has identified an increasing need for an operating system which allows for the transition from research to clinical use. We present eMED-DNA, an in silico operating system for archiving and managing all forms of electronic health records (EHRs) within one's own copy of the sequenced genome to aid in the application and integration of genomic medicine within real world clinical practice. We incorporated an efficient and sophisticated in-DNA file management system for the lossless management of EHRs within a genome. This represents the first in silico integrative system which would bring closer the utopian ideal for integrating genotypic data with phenotypic clinical data for future medical practice.
\end{abstract}

\title{
Introduction
}

The application of DNA as a digital information storage medium has long been considered a viable paradigm, but the real-world impact especially within the confines of actual clinical electronic health care record systems remains an enigma. ${ }^{1-8}$ The initial enthusiasm for such work was to enhance and better assimilate disparate health care information for each patient whilst integrating one's underlying genomic data. Yet, the inherent problem with another form of data acquisition in health systems is the storage and assimilative properties of these often-large data files within the confines of clinical electronic medical systems and electronic health records (EHRs). Furthermore, the integration of genomic data coupled with multiple imaging modalities, consultation and prescription notes means this also poses a difficult task to formulate a robust, secure, accessible and transferable pathway to be applied in real world clinical settings.

In this regard, we demonstrate a simple, robust and informative computational clinical pipeline whereby genomic data across the genome can both be analyzed and simultaneously be integrated with one's other clinical health records; hence providing a seamless archiving system for medical records and clinical genomic data. We have utilized a system in which a copy of the archived patient genome is used, and we particularly use the non-coding regions (introns and intergenic regions) of the human genome, to archive and store electronic health records as a proof of concept, whilst always maintaining the underlying archived genome sequence of the patient for future analysis. Moreover, our system can take any set of genomic locations, provided by a user, which is deemed not useful for a particular clinical context. 
Our system is effective with any form of EHR, e.g., physician's notes, lab reports, various image files which are typically stored in DICOM (Digital Imaging and Communications in Medicine) format. DICOM is the most universal and fundamental standard in digital medical imaging. It defines all the necessary file formats and network protocols to exchange the medical data. We selected computer files to be encoded as a proof of concept for practical DNA-storage, choosing a range of common medical formats to emphasize the ability of our system to store arbitrary digital information.

Given the variable spatio-temporal areas of non-coding regions across the human genome, which posed difficulty during the storage of files which span greater than the largest continual noncoding regions, we have innovated a sophisticated file management operating system that first encodes an EHR into DNA bases and then split it into chunks so that these chunks can be stored across various defined regions in the human genome. However, these regions will not affect analysis or interpretation of one's genome given this is all done in a copied version, whilst archiving the original genome sequence. In particular, our contribution entails:

- An efficient in-DNA file management system ( $i \mathrm{DFMS})$.

- A novel technique for converting binary files to DNA sequences which is specially tailored for the binary data stream resulting from medical imaging files, but is suitable for any binary file. Our proposed encoding is more compact and better than the traditional binary to DNA mapping used in DNA storage.

- Analyzing and identifying various compression techniques appropriate for archiving EHRs.

- Introducing several concepts from operating systems (e.g., virtual memory, abstract memory space, dictionary for storing meta-data, etc.) to the domain of genome sequence, and DNA storage.

- Introducing efficient encoding (binary to DNA bases) and placement strategies for the inDNA dictionary entries.

All these techniques are seamlessly combined into an integrated pipeline eMED-DNA for random and error free management of EHRs within a genome sequence. We believe that our system will facilitate the medical practitioners in managing and transferring both genomic and clinical phenotypic data across different medical and research institutes, and pave the way for meaningful integration of genotypes and phenotypes for precision medicine. Moreover, the new binary-to-DNA mapping strategy and in-DNA file management system, that we have introduced, will contribute towards the improvement of DNA storage technologies. We implemented eMEDDNA as a portable and cross-platform proof of concept software, which is publicly available at https://github.com/jakariamd/eMED-DNA. A comprehensive step-by-step video tutorial is available at https://jakariamd.github.io/eMED-DNA/.

\section{Results}




\section{Archiving EHRs into DNA sequence}

In this section, we provide a brief overview of eMED-DNA and some of its key components. We refer to the Methods section and supplementary materials SM1 for additional details of eMEDDNA and various algorithms used in it. Our pipeline (shown in Fig. 1) starts with compressing EHR components (e.g., physicians' notes, DICOM files, bills, etc.) using appropriate compression techniques. For greater compression ratio, we explored various compression techniques for various types of EHR files and proposed customized compression techniques for DICOM images as these are the most space consuming components of EHRs. Next, binary bits of these compressed files are mapped to DNA base sequence $\{\mathrm{A}, \mathrm{T}, \mathrm{C}, \mathrm{G}\}^{*}$. The naive and the simplest way to convert a binary sequence $B=\{0,1\}^{*}$ to a DNA base stream $D=\{A, T, C, G\}^{*}$ is to encode two bits of B into one bit of D. Suppose, one can encode 00 to A, 01 to T, 10 to C, and 11 to $\mathrm{G}$. Another approach is phase-change encoding which takes the run-length of consecutive 0 's and 1's into account, and was used in the Microvenus project. ${ }^{10}$ In run-length encoding, runs of 0's and 1's are stored as a single data value and count, rather than as the original run. We developed a new technique, especially tailored for DICOM files but suitable for any binary data, with improved performance compared with the trivial one which encodes two binary bits into a particular DNA base (see Methods section for full details of this encoding technique). Experiments on a CT (computerized tomography) scan with 262 DICOM files show that our method substantially improves upon the trivial mapping and saves more than one hundred and seventy thousand DNA bases for this CT scan (see Sec. 1.2 and Fig. S4 in supplementary materials SM1). The resultant DNA stream is subsequently compressed for further space efficiency.

Next, we store the resultant DNA bases in patient's genome sequence. We divide the DNA streams into smaller chunks so we can spread them over the non-coding regions of the genome. We maintain an in-DNA dictionary for indexing the saved files and their positions in the genome so we can retrieve the stored files correctly. The dictionary itself is converted into DNA sequence and stored in the genome. It is consequently necessary to differentiate between DNA bases corresponding to EHRs from those of dictionary entries. Thus, we have to store and manage the dictionary efficiently so we do not lose any meta-data and at the same time we have to make sure it does not grow and overwrite the contents of an EHR which has already been written into the DNA sequence. For optimization and similar to the concept of the stack in random access memory (RAM), we write the dictionary in a reverse direction (relative to EHRs) starting from the end of the genome and progressing towards the front end (i.e., from right to left), whereas the EHR files are saved from the beginning of the genome (Fig. 3). See supplementary materials for full details. 


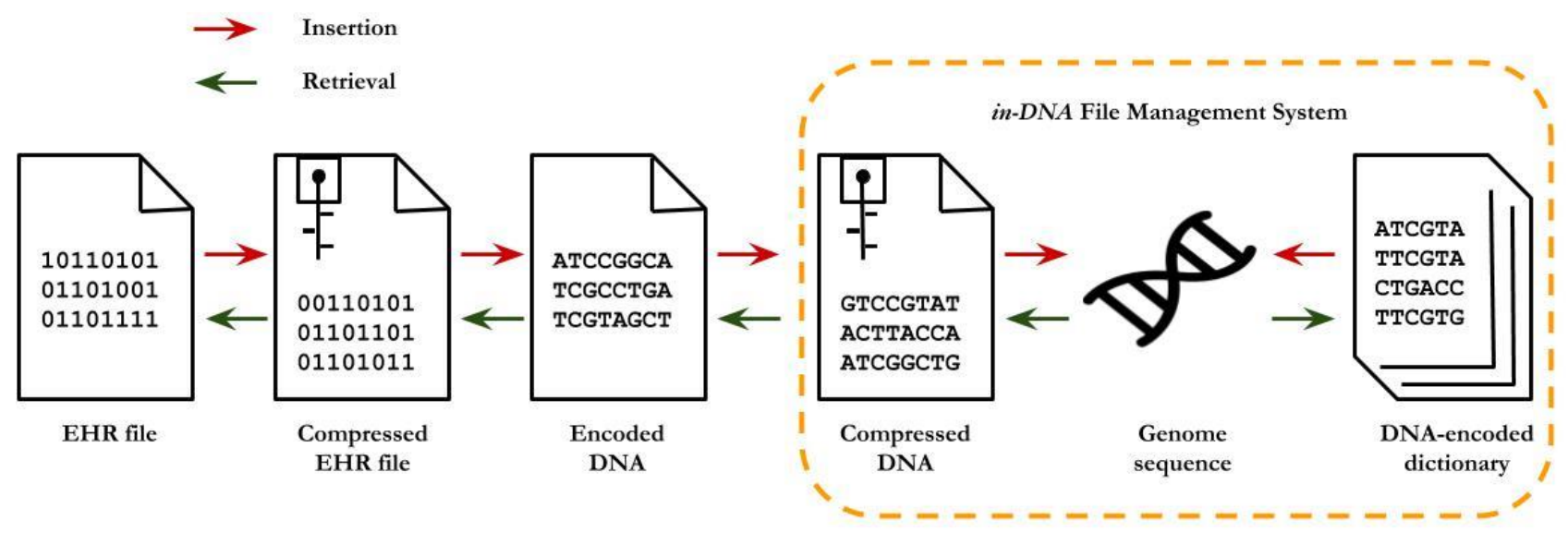

Figure 1: Overview of eMED-DNA. We first compress the EHRs with appropriate compression techniques. Next, the compressed binary files are mapped to DNA base sequence using our proposed binary to DNA base coversion technique. We further compress the DNA base stream, and finally we store the resultant DNA base stream within the non-coding regions (alternatively, the genomic regions provided by the user) of the patient's genome. In addition to the EHRs, we encode the dictionary entries containing the meta-data into DNA bases and store them in the whole genome. The in-DNA file management system provides a random access and lossless architecture for archival and retrival of the data in a genome sequence.

eMED-DNA takes a human genome as an input. A reference human genome GRCH38 (Genome Reference Consortium Human Build 38), obtained from ENSEMBL, was used in our proof-ofconcept. eMED-DNA also takes two other input files - one containing the locations (start and end markers) of the genes in chromosomes to identify the intergenic regions and the other containing the numbers of nucleotide bases in each chromosome. eMED-DNA can detect if any file has previously been saved in the given genome and shows the users a list of the saved files. A user can select, from the list, a filename to decode, so as to view the EHR or save it outside of the genome. eMED-DNA allows inserting new EHRs, as well as deleting the existing ones. The dictionary and the meta-data are updated accordingly after each operation.

\section{in-DNA File Management System (iDFMS)}

In this section, we briefly discuss various key components of our proposed $i$ DFMS. Non-coding regions are of various lengths and are distributed across the whole genome. As already mentioned, a stored EHR file may span several non-coding regions (fully or partially), and may span over more than one chromosome.

The deletion of a stored file from the DNA sequence will make the scenario even more complicated as it will free some regions in the DNA sequence which we have to mark for future 
use. To handle these challenges in a reasonably simpler way, we virtually assemble the noncoding locations from all chromosomes of a genome to form an abstract continuous space. We call this abstract space the free genome space (see Fig. 2).

Coding region

Non-coding region

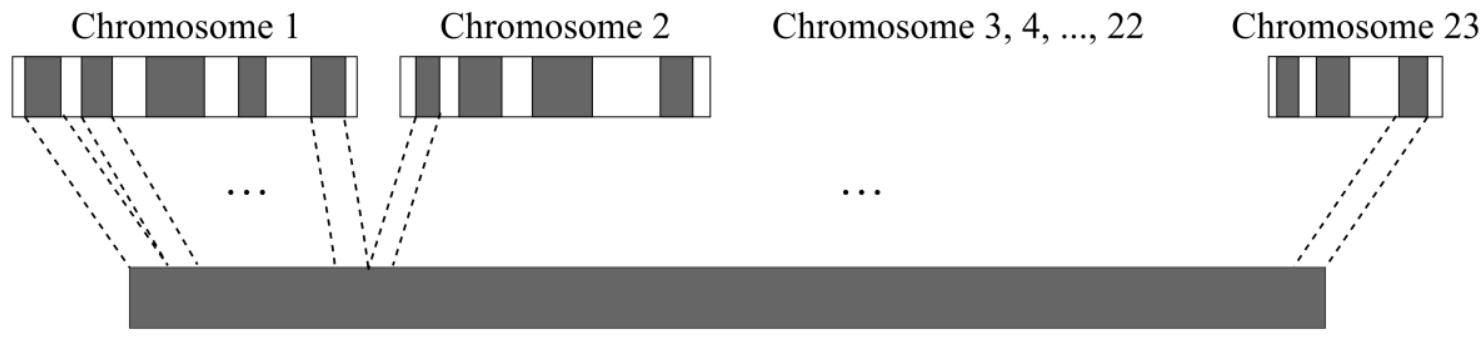

Abstract Continuous Storage

Figure 2: Abstract continuous space in a genome. eMED-DNA virtually assembles the noncoding regions (alternatively, it can assemble any set of genomic locations provided by the user to store EHRs) to an abstract continuous space.

We denote by a user session the time span, after entering a valid input set to the eMED-DNA system, for which the system remains actively open for that input set. At the beginning of every user session, our system constructs the corresponding abstract continuous storage space and creates a map between locations of abstract space and actual chromosome numbers and positions. In this way, a virtual abstract space (free genome space) is created in the beginning of a user session, resulting into simple and elegant file management without having to deal with complicated calculations for every file operations. While performing various file operations (insertion/deletion/retrieval), these abstract locations are converted to actual genomic positions appropriately.

\section{in-DNA Dictionary}

We introduced an in-DNA dictionary in our $i$ DFMS for storing various types of meta data. For each EHR file, we create an entry in the in-DNA dictionary which consists of the following four attributes: name of the file, type (DICOM or non-DICOM), transfer syntax and genomic locations containing the DNA bases of this particular file. When a genome is given as input, eMED-DNA can track the files (if any), which have already been saved in this genome. We have developed a new lossless mapping process to convert a dictionary to DNA base sequences 
(described in Methods) which is completely reversible so that it can decode the dictionary entries corresponding to the files that have already been stored in this genome in previous user sessions. We store the DNA bases resulting from the in-DNA dictionary in a direction which is opposite to the direction of storing DNA bases resulting from the EHRs (as shown in Figure 3). Please see Methods and Secs. 1.4 and 1.5 in supplementary materials SM1 for full details.

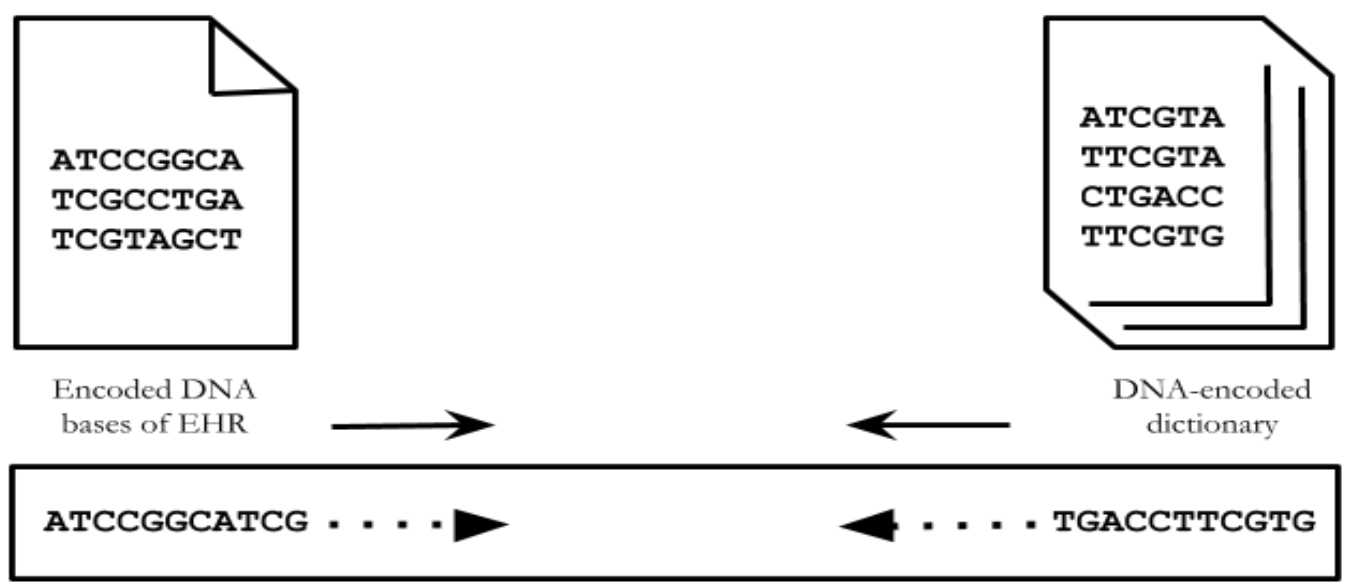

Abstract continuous space (free genome space)

Figure 3: Placement of the EHR files and dictionary entries in a genome. We write the DNA bases resulting from EHRs from the beginning of the free genome space, as opposed to the inDNA dictionary which starts from the end and grows towards the front end of a genome.

To emphasize the effectiveness of eMED-DNA in handling arbitrary digital information, we stored various types of EHRs using eMED-DNA and the summary statistics are provided in Table 1.

Table 1: Summary statistics of the various types of files stored using eMED-DNA. We choose various types of files to demonstrate the ability of eMED-DNA in handling any form of EHRs. These files are anonymized and available at https://git.io/fhIDE.

\begin{tabular}{|l|l|l|l|l|}
\hline File name & File type & File size & $\begin{array}{l}\text { Number of bases } \\
\text { in the encoded } \\
\text { DNA stream }\end{array}$ & $\begin{array}{l}\text { No of chunks } \\
\text { required }\end{array}$ \\
\hline $\begin{array}{l}\text { MR-MONO2-8- } \\
16 x-h e a r t . d c m\end{array}$ & $\begin{array}{l}\text { Dicom (Magnetic } \\
\text { Resonance Imaging) }\end{array}$ & $10,49,496$ & $16,95,640$ & 22 \\
\hline $\begin{array}{l}\text { US-PAL-8-10x- } \\
\text { echo.dcm }\end{array}$ & $\begin{array}{l}\text { Dicom } \\
\text { (Echocardiogram) }\end{array}$ & $4,83,610$ & $21,04,304$ & 71 \\
\hline
\end{tabular}




\begin{tabular}{|l|l|l|l|l|}
\hline Clinical note & $\begin{array}{l}\text { Non-dicom } \\
\text { (physician's notes) }\end{array}$ & 2,264 & 5,060 & 2 \\
\hline
\end{tabular}

Table 2 shows the distribution of the encoded DNA base streams over various chunks of noncoding regions for a single EHR file. See Sec. 1.7 in supplementary materials for additional results.

Table 2. Distribution of the encoded DNA base streams of a sample DICOM file over various non-coding regions using eMED-DNA. This MRI file requires 21 chunks of intergenic regions of various sizes to fit the $16,95,640$ encoded bases. The sample file is available at: https://git.io/fhIDq.

\begin{tabular}{|c|c|c|c|c|c|c|c|}
\hline \multicolumn{8}{|c|}{ File Name : MR-MONO2-8-16x-heart.dcm (MRI) } \\
\hline \multicolumn{4}{|c|}{ File Size: 1025 KB $(10,49,496$ bytes $)$} & \multicolumn{4}{|c|}{ Encoded DNA Stream Length: $16,95,640$ bases } \\
\hline \multicolumn{8}{|c|}{ Location in Genome (21 chunks) } \\
\hline $\begin{array}{c}\text { Chunk } \\
\text { No }\end{array}$ & $\begin{array}{c}\text { Chromosome } \\
\text { No }\end{array}$ & $\begin{array}{c}\text { Chunk } \\
\text { Start } \\
\text { Position }\end{array}$ & $\begin{array}{c}\text { Chunk } \\
\text { Size }\end{array}$ & $\begin{array}{c}\text { Chunk } \\
\text { No }\end{array}$ & $\begin{array}{c}\text { Chromosome } \\
\text { No }\end{array}$ & $\begin{array}{c}\text { Chunk Start } \\
\text { Position }\end{array}$ & $\begin{array}{c}\text { Chunk } \\
\text { Size }\end{array}$ \\
\hline 1 & 1 & 4332003 & 322729 & 12 & 1 & 6579758 & 243 \\
\hline 2 & 1 & 4792535 & 1070275 & 13 & 1 & 6589281 & 1443 \\
\hline 3 & 1 & 6101194 & 599 & 14 & 1 & 6624034 & 832 \\
\hline 4 & 1 & 6180124 & 1145 & 15 & 1 & 6701925 & 83399 \\
\hline 5 & 1 & 6235973 & 8219 & 16 & 1 & 7769707 & 1562 \\
\hline 6 & 1 & 6245579 & 1767 & 17 & 1 & 7781433 & 2887 \\
\hline 7 & 1 & 6260976 & 3293 & 18 & 1 & 7853513 & 62381 \\
\hline 8 & 1 & 6394392 & 18026 & 19 & 1 & 7943166 & 11125 \\
\hline
\end{tabular}




\begin{tabular}{|c|c|c|c|c|c|c|c|}
\hline 9 & 1 & 6424671 & 117 & 20 & 1 & 7985506 & 18898 \\
\hline 10 & 1 & 6520062 & 1285 & 21 & 1 & 8026309 & 84770 \\
\hline 11 & 1 & 6554536 & 645 & & & & \\
\hline \multicolumn{8}{|c|}{ in-DNA Dictionary Entry } \\
\hline \multirow{2}{*}{\multicolumn{2}{|c|}{ Length: 129 bases }} & \multirow{2}{*}{\multicolumn{2}{|c|}{$\begin{array}{c}\text { Location in } \\
\text { Genome ( } 1 \text { chunk) }\end{array}$}} & \multicolumn{2}{|c|}{ Chromosome No } & $\begin{array}{c}\text { Chunk Start } \\
\text { Position }\end{array}$ & $\begin{array}{l}\text { Chunk } \\
\text { Size }\end{array}$ \\
\hline & & & & \multicolumn{2}{|c|}{ Y chromosome } & 57227181 & 129 \\
\hline
\end{tabular}

\section{Discussion}

Due to the advent of next generation sequencing within real world clinical medicine scenarios and the boom of the electronic health record systems to democratize personal health information, there has been an ongoing drive to integrate genomic data into clinical pathways. ${ }^{11-14}$ The "All of Us" research program, formerly known as Precision Medicine Initiative, is expected to generate genomic data, in combination with electronic health records and participant-reported data, from approximately one million US residents with diverse backgrounds, so as to better risk stratify patients and enhance personalized diagnostics. ${ }^{15,16}$

Precision medicine emphasizes the need for integrating genomic data and patient's health records. ${ }^{17,18}$ However, including genomic sequences in EHRs has raised some complexities. Apart from security and ethical issues, there are practical challenges to integrate genomic data into electronic health records which include size and complexity of genetic test results, inadequate use of standards for clinical and genetic data, and limitations in EHR's capacity to store and analyze genetic data. ${ }^{19}$ Furthermore, the plethora of large scale genomic studies has led to the discovery of many putative genes for better risk stratifying and enhancing diagnostic decision trees. ${ }^{19-23}$ Therefore, various large-scale initiatives have been taken for translating insights from genomics into clinical medicine. ${ }^{11-14,23}$ Hence, the efficient management, organization and protection of both genomic data and clinical records will be a greater necessity. eMED-DNA is a unique system for the delivery of genomic and clinical care in one operating system.

The eMED-DNA system not only converts generic file formats such as prescriptions, clinical lab results and physician reports, it also deals with clinical image files such as DICOM files and converts them into DNA sequences within the genome. This workflow can also be extended whereby these converted files can be synthesized into oligonucleotides and archived for a long period of time. Apart from the evident benefit in archiving, managing and transferring heterogeneous medical data across various medical and research institutes, this will have a direct impact in various retrospective medical studies. Although we did not perform any experimental 
studies to synthesize the genome sequence (obtained by eMED-DNA) into artificial DNA, existing DNA storage techniques can easily be coupled with eMED-DNA to facilitate artificial DNA synthesis. ${ }^{8-9}$ Therefore, although we customized our encoding technique as an in silico system to avoid particular forms of repetitions, our system does not explicitly consider various issues associated with physical DNA storage, such as, repeats, homopolymers, small reads, etc. For example, Goldman et al. avoided repeats by translating the binary computer information into ternary number system and then encode the information into DNA bases. ${ }^{5}$ Yet, it is plausible that our in silico digital system can be part of a means to also identify and synthesise various EHR data for long term storage. Our proof-of-concept currently does not explicitly consider any privacy issue associated with genomic data sharing. However, the human genome can reveal sensitive information and is vulnerable to re-identifiability risk. ${ }^{16,24-30} \mathrm{We}$ believe a more sophisticated pipeline, which does not overwrite any region of the genome sequence such as ours, is more desirable as future research may reveal relevance of other regions which is currently considered as "junk" DNA. Indeed, recent research suggested that there may be relationships between introns and proteins, and long intergenic non-coding RNAs (lincRNAs) have been shown to have important functionalities. ${ }^{31-32}$ With a more sophisticated mapping system between EHRs and DNA bases, eMED-DNA would be able to retrieve the genomic regions, that it uses for storing EHRs, without having to keep an extra copy of the genome. (See Sec. 2 in supplementary materials for additional discussion).

The timing of this approach seems appropriate as obtaining genomic data are becoming easier and cheaper, DNA storage techniques are getting better which leads many to speculate on its near-term potential as a practical storage media ${ }^{5}$, population level genetic variation and understanding the genetic basis of diseases are getting significant attention from the research community ${ }^{33-35}$, and finally we are witnessing a rapid acceleration in the use of genomic information in patient care. We believe our proof-of-concept platform is the first of its kind and can greatly facilitate the future of precision medicine.

\section{Methods}

\section{Encoding Binary files to DNA sequence}

The naive and the simplest way to convert binary sequence $\mathrm{B}=\{0,1\}^{*}$ to DNA base stream $\mathrm{D}=$ $\{A, T, C, G\}^{*}$ is to encode two bits of B into one bit of D. Suppose, one can encode 00 to A, 01 to $\mathrm{T}, 10$ to $\mathrm{C}$, and 11 to $\mathrm{G}$. Another approach is phase-change encoding which takes the run-length of consecutive 0 's and 1's into account, and was used in the Microvenus project. ${ }^{36}$ In run-length encoding, runs of 0's and 1's are stored as a single data value and count, rather than as the original run. 
We developed a novel binary to DNA base conversion approach especially targeted for binary streams resulting from medical imaging files (DICOM). We observed the presence of long runs of 0's in the binary streams of DICOM files which would be converted into long sequence of A's in the usual encoding. Such repetitions are problematic for synthesizing, and are more likely to be misread by DNA-sequencing machines, leading to errors when reading the information back. $^{37}$

Although homopolymers of short length does not make much problem in Illumina sequencing platforms, sequences of high or low GC content is difficult to sequence correctly. ${ }^{1}$ And higher frequency of A's, resulting from long streams of 0's, will decrease the GC content of the entire string.

Although we observed the presence of long runs of 0's in the binary stream of DICOM files, the frequency of shorter runs of 0's was much higher than the longer runs. For shorter runs of 0's, the run-length based approaches give poorer compression than the naïve encoding. So we came up with an approach that does the naive encoding for shorter runs and run-length encoding for larger runs. We introduced a hyper parameter, which we call 'shift', to serve this purpose. We do not take the long runs of 1's into account as they are not usually prevalent in the binary streams of DICOMs due to their particular structures and grayscale values (see Figure S1 in supplementary materials SM1).

We read the binary bit stream by 2 bits at a time as the number of bits is always even $(1$ byte $=8$ bits). We denote by run-length the length of a run of consecutive 0's. If the run-length >= shift +2 , we use run-length encoding; otherwise we use the naive encoding technique. Therefore, during the decoding step, we need to correctly differentiate the run-length encoded base streams from the base streams that have been encoded using the naive mapping. We use a prefix comprising a number of consecutive A's equal to shift/2 +1 to mark the start of run-length encoding, and a single ' $A$ ' as a suffix to denote the end of the run. We store the run-length information between the prefix and the suffix by encoding the value of difference. We propose a new mapping technique to encode difference using DNA bases. Since 'A' is used as a suffix for the run-length information, the run-length information must be encoded with a ternary code that uses the remaining DNA symbols ( $\mathrm{C}, \mathrm{G}$ and $\mathrm{T})$. One way to encode the difference would be converting difference/2 to ternary numbers (base 3 ), and finally mapping each of the base 3 digits $(0,1$ and 2$)$ to a different DNA base (T, C and G). However, by encoding difference using this approach, ternary numbers starting with 0 (e.g, 0 (T), 00 (TT), 000 (TTT), 010 (TCT), etc.) will never appear. In general, for $n$ bits we should be able to use $3 n$ permutations, but using simple base 3 conversion would only utilize $2 * 3^{\text {n-1 }}$ permutations. Therefore, we have developed an efficient conversion technique (shown in Table S2 in supplementary materials SM1) which would utilize all possible permutations of the available three symbols, and thereby require fewer bits than the simple base 3 encoding described above. 
Our technique is an incremental mapping between DNA bit sequence and the value of difference. It is designed in a way that enables us to represent run-lengths using minimum number of DNA bits possible. We developed a DNA base based number system accordingly. Suppose, 'T', 'C', and ' $\mathrm{G}$ ' represent 1, 2 and 3, respectively. Then the numbers using only these characters are as follows: 1 (T), 2 (C), 3 (G), 11 (TT), 12 (TC), 13 (TG), 21 (CT), 22 (CC), and so on. The value of difference starts with 2 and is increased by 2 as we read two DNA bases at a time. Note that there is no DNA base mapping for the starting value (2) of difference. See supplementary materials SM1 for additional details.

\section{Converting the dictionary to DNA bases}

For each stored file, we create an entry in the in-DNA dictionary which comprises the following attributes:

- Name: name of file $(f)$.

- Type $(t)$ (DICOM or non-DICOM): to select decompression procedure based on the type of the files.

- Transfer Syntax (s) (implicit or explicit): this is relevant only for DICOM files as lossless DICOM file decompression using j2k method requires transfer syntax.

- Genomic locations $(L)$ : a sorted list of all the chunks (with respect to the starting positions) which contain the sequence data of a particular file.

The dictionary of a genome itself is stored in the genome along with the actual files so that we do not have to manage separate files for meta data. After encoding a dictionary entry to a DNA base stream and storing it to the genome sequence, we need to be able to distinguish between various components (e.g, name, type, transfer syntax, genomic locations etc.) so we can read them back and decode the dictionary entry correctly. Therefore, we put some special markers in between these components. Note that these markers have to be encoded as DNA bases as well. DNA bases used to encode the markers should consequently be distinguishable from the encoded DNA base streams of the various dictionary components. Moreover, markers may be required between different dictionary entries to enable identification and reading of the individual entries correctly. Considering all these challenges, we have designed an efficient dictionary to DNA base stream conversion procedure which allows us to distinguish between individual dictionary entries and their components so we can decode them without any loss of information and ambiguity.

For a dictionary entry $e$ with four components $(f, t, s, L)$, we write them (starting from the tail end of the genome and progressing towards the front end) in the following order: $f, s, L$ and $t$. We first describe the encoding technique of the file name $(f)$ and the transfer syntax $(s)$. The first 32 ASCII characters (out of 256) never appear in a file name as they are various control characters. 
These 32 characters have 0's in the three most significant positions in an 8-bit binary representation, which allows us to use " 000 " as a part of a marker. We add one more bit to incorporate the transfer syntax as well. That means, we use 0000 (AA) to denote the end of the file name as well as to denote the implicit transfer syntax, and use 0001 (AT) to denote the end of a file name and explicit transfer syntax. These two markers will not appear in the DNA base streams of the file names, and can be used to not only mark the end of the file name, but denote the transfer syntax as well.

We now describe the process for encoding $L$ (genomic locations) and $t$ (file type) of a dictionary entry. Let $L$ be a set $\left\{c_{1}, c_{2}, c_{3}, \ldots, c_{\mathrm{k}}\right\}$ of chunks in the genome sequence that make up a particular EHR. Here a chunk $c_{i}$ is identified by its start position $\left(n_{1}\right)$ and size $\left(n_{2}\right)$. Note that $n_{1}$ and $n_{2}$ are integers and so they do not contain any character other than $0 \sim 9$.

We represent each digit $(0 \sim 9)$ by four bits and encode them into DNA base pairs as shown in Table S1 in SM1. We encode 00 by A, 01 by T, 10 by $\mathrm{C}$ and 11 by G. As already mentioned, the encoding to DNA bases is from right to left as as dictionary entries are written from the tail end of the genome, progressing towards the front. For a number $n=d_{\mathrm{k}} d_{\mathrm{k}-1} \ldots d_{2} d_{1}$ with $k$ digits, we start encoding and writing the digits into the genome sequence from the most significant bit $\left(d_{\mathrm{k}}\right)$ and progress towards the least significant bit $\left(d_{1}\right)$. The corresponding DNA base pairs (as shown in Table 3) are appended from right to left. In this way, as the symbols are read back from right to left, they will be decoded in the appropriate order. For example, let $n=381$. This will be encoded (starting from the most significant bit) as "TAACGA". Therefore, while reading them back from right to left, we will have "AGCAAT" which will be decoded to 381 (considering two characters at a time) according to the example mapping.

Table 3: Mapping of numerical digits to DNA bases in dictionary to DNA base encoding. The base corresponding to the left 2 bits goes to the right. For example, for the digit 2, binary bits are 0010. Left 2 bits (00) map to 'A' and the right 2 bits (10) map to ' $C$ '. Now 'A 'goes to the right of ' $\mathrm{C}$ ', i.e. final 2-length base string for digit 2 is ' $\mathrm{CA}$ '.

\begin{tabular}{|l|l|l|}
\hline Digit & Binary & Base \\
\hline 0 & 0000 & AA \\
\hline 1 & 0001 & TA \\
\hline 2 & 0010 & CA \\
\hline 3 & 0011 & GA \\
\hline 4 & 0100 & AT \\
\hline 5 & 0101 & TT \\
\hline 6 & 0110 & CT \\
\hline 7 & 0111 & GT \\
\hline 8 & 1000 & AC \\
\hline 9 & 1001 & TC \\
\hline
\end{tabular}


There are $2^{4}=16$ possible combinations of length four, of which only 10 combinations have been used in representing ten digits (see Table S3). The six remaining combinations: 1010 (CC), 1011 (GC), 1100 (AG), 1101 (TG), 1110 (CG), and 1111 (GG) may be used for marking the boundaries between various components and individual dictionary entries. Note that no digit in Table 3 starts with a ' $G$ ' (from right to left), and therefore we use ' $G$ ' as a separator to mark the end of a number $n$.

There are two combinations - 1010 (CC) and 1011 (GC) - that are unused in the encoded DNA base stream of $L$. We utilize these two combinations to mark the end of an entry $e$ as well as to denote the file type $t$ (DICOM and non-DICOM). For a DICOM file, CC may be used to mark the end of L, and GC may be used for non-DICOM files. Thus, by carefully designing the encoding techniques for various components of a dictionary entry and defining the order of encoding them, it is possible to distinguish between different components of a dictionary entry as well as the boundaries between individual entries without any ambiguity and loss of information. Figure 4 shows an example of a dictionary entry and the corresponding DNA base stream resulted from our encoding technique.

\begin{tabular}{|l|l|}
\hline \multicolumn{2}{|c|}{ Dictionary Entry } \\
\hline File name & A.dcm \\
\hline File type & Dicom \\
\hline Transfer syntax & Implicit \\
\hline Genomic locations & $\{(30,9),(50,13)\}$ \\
\hline
\end{tabular}

(a)

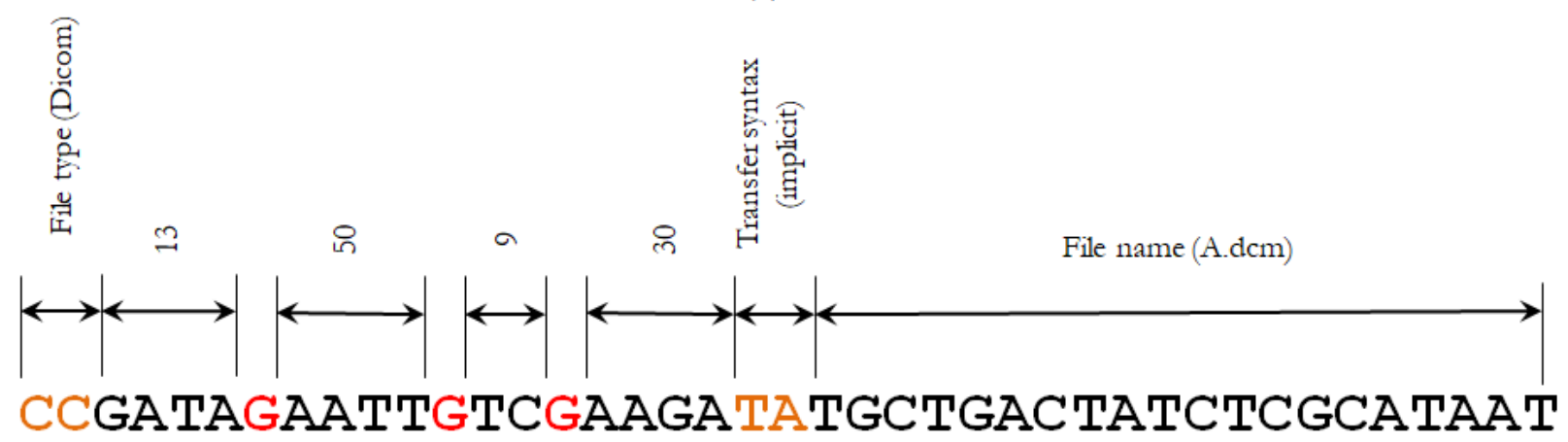

(b)

Figure 4: Encoding of a dictionary entry to DNA bases. (a) A dictionary entry with four components (name, type, transfer syntax and genomic locations). (b) Encoded DNA bases of this entry. We use 0001 (AT) to denote the end of the file name as well as the implicit transfer 
syntax, and CC is used to denote the end of the entry as well as the file type. In the DNA base stream of genomic locations $(L)$, the G's that are in the odd positions (marked in red) serve as markers to separate two numbers.

\section{Data Availability}

Data supporting the findings of this study are available at https:/git.io/fhIDE.

\section{Code Availability}

The eMED-DNA software is freely available at https:/github.com/jakariamd/eMED-DNA. The code is available on request from the authors.

\section{References}

1. Cox JP. Long-term data storage in DNA. Trends Biotechnol 2001;19:247-250.

2. Clelland CT, Risca V, Bancroft C. Hiding messages in DNA microdots. Nature 1999;399:533534.

3. Ailenberg M, Rotstein O. An improved Huffman coding method for archiving text, images, and music characters in DNA. Biotechniques 2009;47:747-754.

4. Church GM, Gao Y, Kosuri S. Next-generation digital information storage in DNA. Science 2012;337:1628.

5. Goldman N, Bertone P, Chen S, et al. Towards practical, high-capacity, low-maintenance information storage in synthesized DNA. Nature 2013;494:77-80.

6. Carr PA, Church GM. Genome engineering. Nat Biotechnol 2009;27:1151-1162.

7. Gantz J, Reinsel D. Extracting value from chaos. IDC iview 2011;1142:1-12.

8. Bornholt J, Lopez R, Carmean DM, Ceze L, Seelig G, Strauss K. A DNA-based archival storage system. Proceedings of the 21th ACM International Conference on Architectural Support for Programming Languages and Operating Systems; 2016 April 2-6; Atlanta, Georgia. ACM New York. NY; 2016. 637-649. 
9. Goldman N, Bertone P, Chen S, et al. Towards practical, high-capacity, low-maintenance information storage in synthesized DNA. Nature 2013;494:77-80.

10. Davis J. Microvenus. Art Journal 1996; 55:70-74.

11. Dewey FE, Grove ME, Pan C, et al. Clinical interpretation and implications of wholegenome sequencing. JAMA 2014;311:1035-1045.

12. Vassy JL, Lautenbach DM, McLaughlin HM, et al. The MedSeq Project: a randomized trial of integrating whole genome sequencing into clinical medicine. Trials 2014;15:85.

13. Manolio TA, Green ED. Leading the way to genomic medicine. Am J Med Genet C Semin Med Genet 2014;166C:1-7.

14. Green ED, Guyer MS; National Human Genome Research Institute. Charting a course for genomic medicine from base pairs to bedside. Nature 2011;470:204-213.

15. Collins FS, Varmus H. A new initiative on precision medicine. N Engl J Med 2015;372:793795.

16. Wang S, Jiang X, Tang H, et al. A community effort to protect genomic data sharing, collaboration and outsourcing. NPJ Genome Med 2017;2:33.

17. Choi M, Scholl UI, Ji W, et al. Genetic diagnosis by whole exome capture and massively parallel DNA sequencing. Proc Natl Acad Sci 2009;106:19096-19101.

18. Ashley EA, Butte AJ, Wheeler MT, et al. Clinical assessment incorporating a personal genome. Lancet 2010;375:1525-1535.

19. Worthey EA, Mayer AN, Syverson GD, et al. Making a definitive diagnosis: successful clinical application of whole exome sequencing in a child with intractable inflammatory bowel disease. Genet Med 2011;13:255-262.

20. Collins FS, McKusick VA. Implications of the Human Genome Project for medical science. JAMA 2001;285:540-544.

21. Ng SB, Turner EH, Robertson PD, et al. Targeted capture and massively parallel sequencing of 12 human exomes. Nature 2009;461:272-276.

22. Bainbridge MN, Wiszniewski W, Murdock DR, et al. Whole-genome sequencing for optimized patient management. Sci Transl Med 2011;3:87re3.

23. Johnson JA, Roden DM, Lesko LJ, Ashley E, Klein TE, Shuldiner AR. Clopidogrel: a case for indication-specific pharmacogenetics. Clin Pharmacol Ther 2012;91:774-776.

24. Bryc K, Durand EY, Macpherson JM, Reich D, Mountain JL. The genetic ancestry of African Americans, Latinos, and European Americans across the United States. Am J Hum Genet 2015;96:37-53.

25. Ashley EA. The precision medicine initiative: a new national effort. JAMA 2015;313:21192120.

26. Homer N, Szelinger S, Redman M, et al. Resolving individuals contributing trace amounts of DNA to highly complex mixtures using high-density SNP genotyping microarrays. PLoS Genet 2008;4:e1000167.

27. Sweeney L, Abu A, Winn J. Identifying participants in the personal genome project by name (a re-identification experiment). arXiv preprint 2013;1304:7605. 
28. Gymrek M, McGuire AL, Golan D, Halperin E, Erlich Y. Identifying personal genomes by surname inference. Science 2013;339:321-324.

29. Harmanci A, Gerstein M. Quantification of private information leakage from phenotypegenotype data: linking attacks. Nat Methods 2016;13:251-256.

30. Wang R, Li Y, Wang X, Tang H, Zhou X, editors. Learning your identity and disease from research papers. Proceedings of the 16th ACM conference on Computer and communications security; 2009 Nov 9-13; Hyatt Regency Chicago, Chicago. ACM New York, 2009 Nov 9. 534-544.

31. Doolittle WF. Is junk DNA bunk? A critique of ENCODE. Proc Natl Acad Sci U S A 2013; 110:5294-5300.

32. Elling R, Robinson EK, Shapleigh B, et al. Genetic Models Reveal cis and trans ImmuneRegulatory Activities for lincRNA-Cox2. Cell Rep 2018; 25:1511-1524.

33. Ashley EA. Towards precision medicine. Nat Rev Genet 2016;17:507-522.

34. Shringarpure SS, Bustamante CD. Privacy Risks from Genomic Data-Sharing Beacons. Am J Hum Genet 2015;97:631-646.

35. Claes P, Liberton DK, Daniels K, et al. Modeling 3D facial shape from DNA. PLoS Genet 2014; 10:e1004224.

36. Davis J. Microvenus. Art Journal 1996; 55:70-74.

37. Niedringhaus TP, Milanova D, Kerby MB et al. Landscape of next-generation sequencing technologies. Anal. Chem. 2011; 83:4327-4341.

Author contributions: R. S and M.S.B conceived the study; M.S.B and R. S. supervised the study; M.S.B., M.J., K.S., M.R.U., M.M.I. and T.D. designed the eMED-DNA pipeline and developed the algorithms used in various components; M.J., K.S., M.R.U., M.M.I. and T.D. implemented eMED-DNA; M.J., K.S., M.R.U., T.D., M.M.I., M.S.B. and R.S. designed and conducted the experiments; M.S.B., R.S., M.J., K.S., M.R.U., T.D. and M.M.I. prepared the manuscript. The first five authors contributed equally and share the first-authorship of this paper. 


\section{Supplementary Materials for}

\section{eMED-DNA: An in silico operating system for clinical medical data storage within the human genome}

These supplementary materials present additional details about various computational techniques and algorithms used in eMED-DNA (Section 1), and also present additional discussion (Section 2). 


\section{TABLE OF CONTENTS}

Section 1 eMED-DNA

Subsection 1.1 Compressing DICOM files

Subsection 1.2 ...... Compressed binary files to DNA sequence

Subsection 1.3 Compressing encoded DNA sequence

Subsection 1.4 Placement of the dictionary in the genome sequence

Subsection 1.6 File Operations

Subsection 1.7 Additional Results

Section 2 Additional Discussion

Figure S1 Histograms showing the frequencies of pixel grayscale intensity values $(0 \sim 255)$ for four sample compressed DICOM files

Figure S2 ....................................... Binary sequence to DNA base stream encoding algorithm

Figure S3. DNA base stream to binary sequence decoding algorithm

Figure S4 Comparison between our encoding technique (with various shift values) and the naive encoding (without shift parameter)

Table S1 A naive binary bits to DNA base $\left(\{0,1\}^{*} \rightarrow\{\text { A, T, C, G }\}^{*}\right)$

encoding .5

Table S2 A novel run-length encoding of binary bits to DNA base sequence conversion .....5

Table S3 Distribution of the encoded DNA base stream of a sample DICOM file over various non-coding regions using eMED-DNA

Table S4 Distribution of the encoded DNA base stream of two clinical notes over various non-coding regions using eMED-DNA

References 15 


\section{1. eMED-DNA}

eMED-DNA is an in silico integrative pipeline which incorporates compression at different levels, a novel Binary-to-DNA base conversion technique, and an in-DNA file management system to manage the DNA base streams resulting from the electronic health records (EHRs) and the corresponding dictionary entries within ones genome sequence. We proposed customized algorithms and computational techniques for each of these key components of eMED-DNA.

\subsection{Compressing DICOM files}

In order to be able to accommodate large scale EHRs, we compress the medical records before encoding them as nucleotide bases. In particular, as DICOM files are the most space consuming and have special file formats, we proposed customized compression techniques suitable for DICOM files. We performed an extensive evaluation study to identify the techniques suitable for DICOM and finally, we customize the existing techniques so that they perform well on medical imaging files.

Compressing medical imaging files is challenging. The American College of Radiology (ACR) does not provide clear recommendation for compression. ${ }^{1}$ The US FDA does not permit compression storage or transmission of breast imaging. For other categories of image, we should carefully choose the type of image compression so that we do not loss any information and do not violate the DICOM standards. So, if there is a viable need for compression due to limited storage capacity, we should stick to lossless compression techniques.

There are many algorithms to compress binary data. But we only tried those which are recommended by DICOM standard. ${ }^{2}$ Current DICOM standard supports RLE $^{3}$ (Run Length Encoding), Deflate ${ }^{4}, \mathrm{JPEG}^{5}, \mathrm{JPEG} \mathrm{LS}^{6}$, and JPEG $2000^{7}$ compression techniques. Among these, JPEG is lossy while RLE, Deflate, JPEG LS and JPEG 2000 are lossless compression techniques. With an extensive evaluation study on various lossless compression techniques recommended by DICOM standards (results not shown), we decided to use JPEG 2000 for our first level of compression, i.e., compressing the binary data of the digital EHR file.

\subsection{Compressed binary files to DNA sequence}

We developed a new technique for encoding binary data to DNA base sequence (described in Methods section of this paper). In this section, we present additional details, examples and supporting experimental results. 

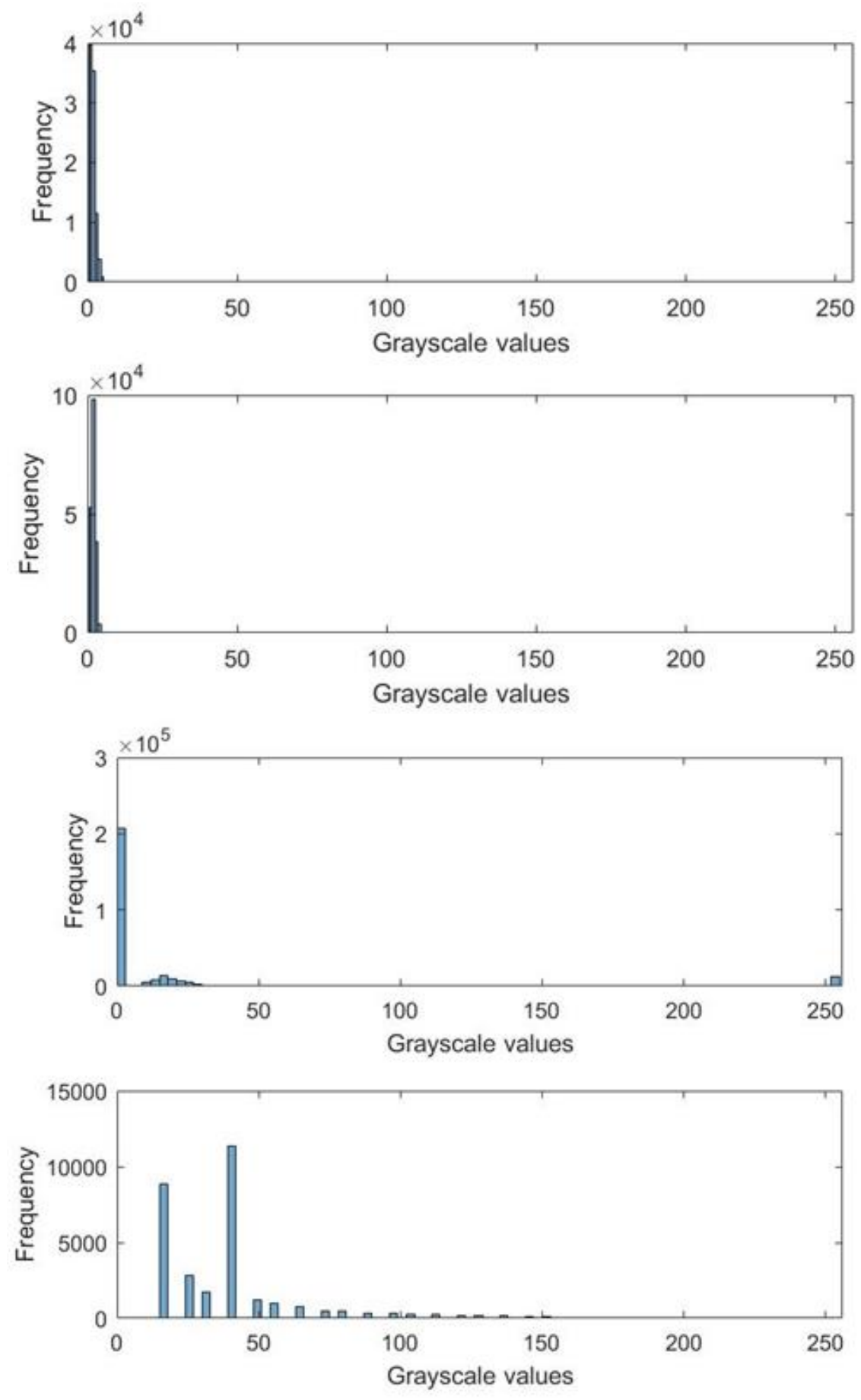

Figure S1: Histograms showing the frequencies of the pixel grayscale intensity values $(0$ 255) for four sample compressed DICOM files (top two plots are for RGB and the bottom two are for monochrome files). These files are available at: https://goo.gl/ruzozJ. All of these plots show right-skewed distribution. The frequencies of smaller values are much higher which reflects the presence of long runs of 0 in the binary stream of the Dicom files. This is due to the larger darker portions in DICOM files which have grayscale value of 0 , i.e., RGB value of $(0,0,0)$. 
Table S1: A naive binary bits to DNA base $\left(\{0,1\}^{*} \rightarrow\{A, T, C, G\}^{*}\right)$ encoding. Each of the four DNA bases can be represented by 2 binary bits. There are $4 !=24$ such naive mappings. The table shows a mapping that we used for encoding compressed (non-DICOM) EHRs and the dictionary entries.

\begin{tabular}{|l|l|}
\hline Bits & Base \\
\hline 00 & $\mathrm{~A}$ \\
\hline 01 & $\mathrm{~T}$ \\
\hline 10 & $\mathrm{C}$ \\
\hline 11 & $\mathrm{G}$ \\
\hline
\end{tabular}

Table S2: A novel run-length encoding of binary bits to DNA base sequence conversion. We show the mapping for values up to 76 . This mapping allows us to utilize all possible $3^{\text {n }}$ permutations of three DNA bases.

\begin{tabular}{|l|l|l|l|l|l|}
\hline DNA bits & $\begin{array}{l}\text { Run length }- \\
\text { shift }\end{array}$ & DNA bits & $\begin{array}{l}\text { Run length }- \\
\text { shift }\end{array}$ & DNA bits & $\begin{array}{l}\text { Run length }- \\
\text { shift }\end{array}$ \\
\hline- & 2 & TTT & 28 & CCC & 54 \\
\hline T & 4 & TTC & 30 & CCG & 56 \\
\hline C & 6 & TTG & 32 & CGT & 58 \\
\hline G & 8 & TCT & 34 & CGC & 60 \\
\hline TT & 10 & TCC & 36 & CGG & 62 \\
\hline TC & 12 & TCG & 38 & GTT & 64 \\
\hline TG & 14 & TGT & 40 & GTC & 66 \\
\hline CT & 16 & TGC & 42 & GTG & 68 \\
\hline CC & 18 & TGG & 44 & GCT & 70 \\
\hline CG & 20 & CTT & 46 & GCC & 72 \\
\hline GT & 22 & CTC & 48 & GCG & 74 \\
\hline GC & 24 & CTG & 50 & GGT & 76 \\
\hline GG & 26 & CCT & 52 & $\ldots$ & $\ldots$ \\
\hline
\end{tabular}

By analyzing the mapping table (Table S2) we can see that if difference $=$ run-length - shift is within the range $\left[3^{n}+1,3^{n+1}-1\right]$, we need $n$ DNA bits to encode this difference value. Our encoding algorithm computes the value of $n$ and converts (difference $-3^{n}+1$ ) $/ 2$ using a base- 3 conversion and encodes the resulting ternary (base-3) number of $n$ digits using a simple mapping 
as follows: $\{0 \rightarrow \mathrm{T}, 1 \rightarrow \mathrm{C}, 2 \rightarrow \mathrm{G}\}$. For example, let the value of difference be 22 . This value (22) is within the range $\left[3^{2}+1,3^{2+1}-1\right]$, meaning two DNA symbols are required to encode difference. A base 3 conversion of $\left(22-\left(3^{2}+1\right)\right) / 2=6$ is $20\left[(6)_{10}=(20)_{3}\right]$. Finally, we map this to DNA bases $(20 \rightarrow \mathrm{GT})$ using the simple mapping mentioned above. Note that a direct base- 3 conversion of this value (22) would require 3 DNA bits $(\mathrm{CTG})$ as $(22 / 2)_{10}=(11)_{10}=(102)_{3}$, whereas our method takes only 2 DNA bits.

This encoding technique not only offers higher storage capacity, it also allows us to design a lossless decoding algorithm. Our decoding technique reads the nucleotide base stream character by character. Unless the conditions for transitioning to run-length decoding are met, each character of the nucleotide sequence is simply mapped to two binary bits. However, in order to monitor for a transition marker, the decoding algorithm checks whether each ' $A$ ' is part of a runlength prefix by checking whether there are enough consecutive 'A's to be a prefix of run-length encoding. If a transition marker/prefix is identified, the DNA bases between the prefix and the suffix are read. Let the number of DNA bases in between the prefix and the suffix be len. The corresponding encoded value can be determined using the mapping $(\mathrm{T} \rightarrow 0, \mathrm{C} \rightarrow 1, \mathrm{G} \rightarrow 2$ ) followed by a base-3 to base-10 conversion. Next, the difference can be calculated as follows: $v a l^{*} 2+\left(3^{n}+1\right)=$ difference. Finally, a number of ' 0 's equal to difference + shift is added to the decoded binary bit stream. Suppose, the string between suffix and prefix is 'GT', then len equals 2 and val equals $(20)_{3}$. After a base-10 conversion, val becomes 6 (since $(20)_{3}=(6)_{10}$ ). So, a number of ' 0 's equal to $6^{*} 2+$ shift $+3^{2}+1$ or $22+$ shift is added to the binary bit stream. The pseudo codes for encoding and decoding are shown in Fig. S2 and Fig. S3, respectively. 


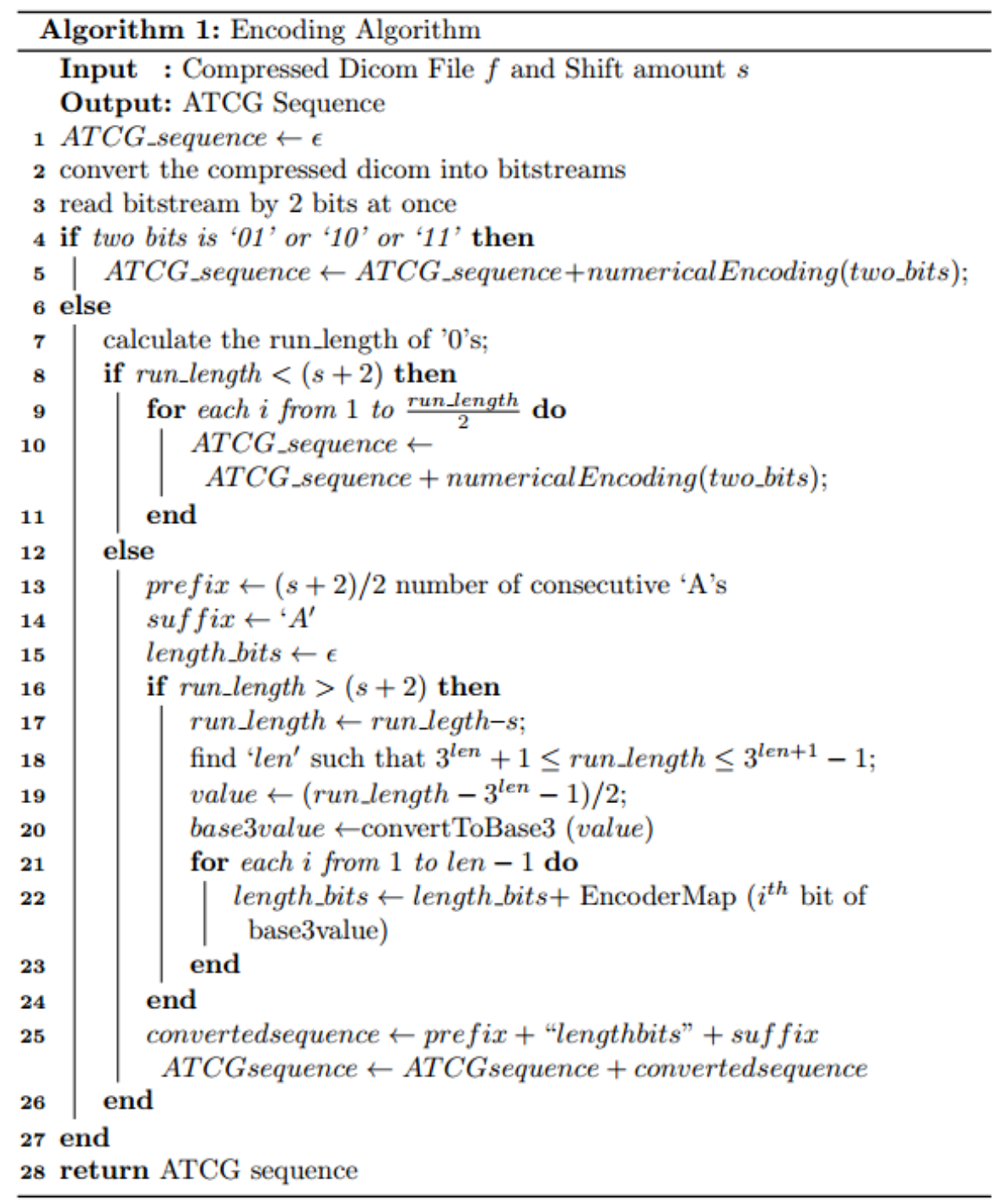

Figure S2: Binary sequence to DNA base stream encoding algorithm. 


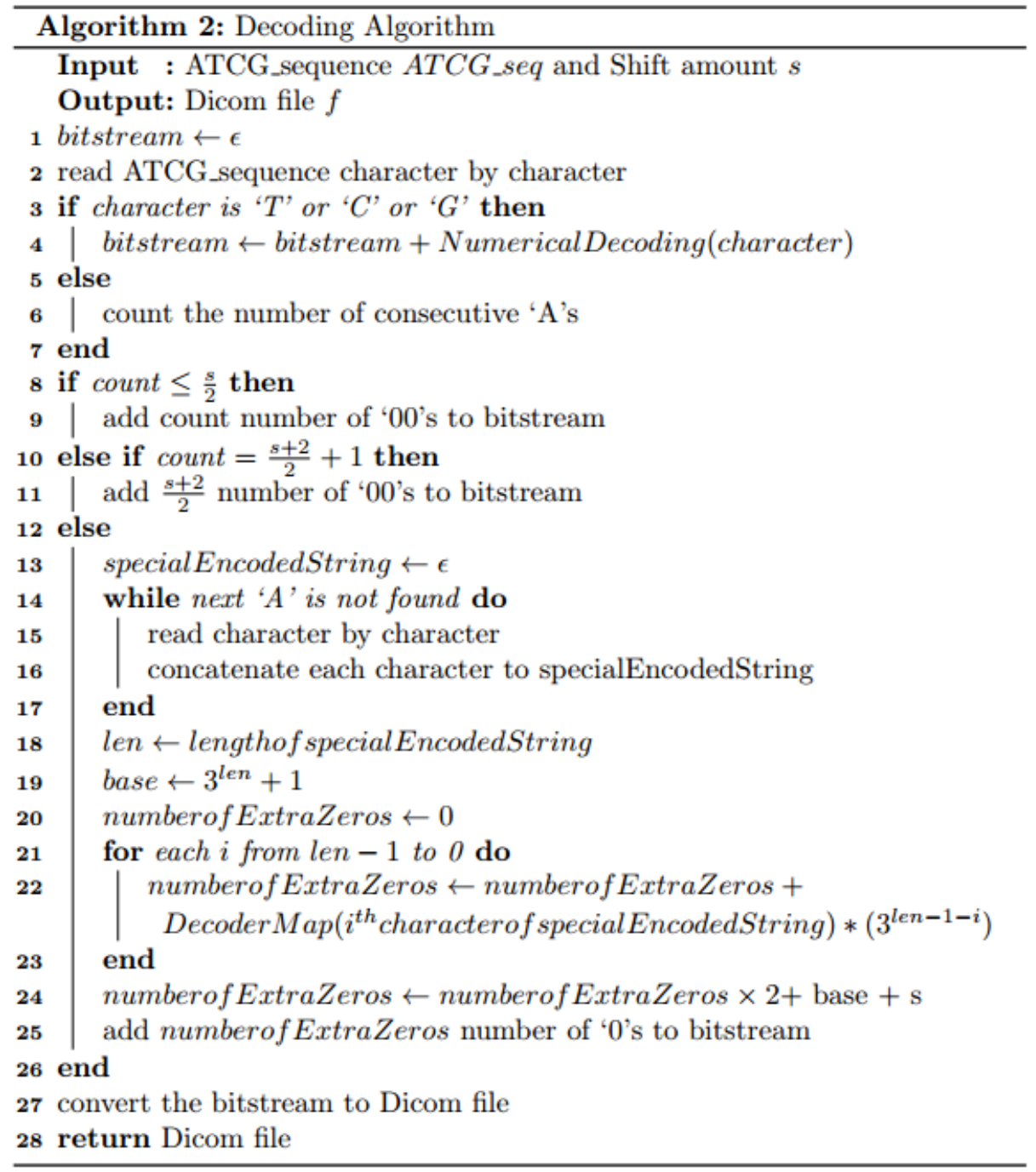

Figure S3: DNA base stream to binary sequence decoding algorithm.

Using this encoding and decoding techniques, we can achieve better compression ratio than the naive approach for most of the DICOMs that we tested. We evaluated the performance of our method on a CT (computerized tomography) scan of the skull consisting of 262 DICOM files and varied the values of the hyper parameter 'shift' (see Fig. S4). The average difference between our technique with shift $=16$ and the naive method is around 650 DNA bases. Therefore, for the single CT scan we analyzed, the difference is around one hundred and seventy thousand DNA bases $(262 * 650=1,70,300)$ which is clearly a substantial improvement. Thus our technique improves upon the naive mapping, especially when there is substantial amount of long runs of 0 's, which makes it suitable for DICOM files like CT scans. Further extensive evaluation on various types of DICOM files is required to better assess the performance of this encoding technique and identify a suitable value for the shift parameter. 


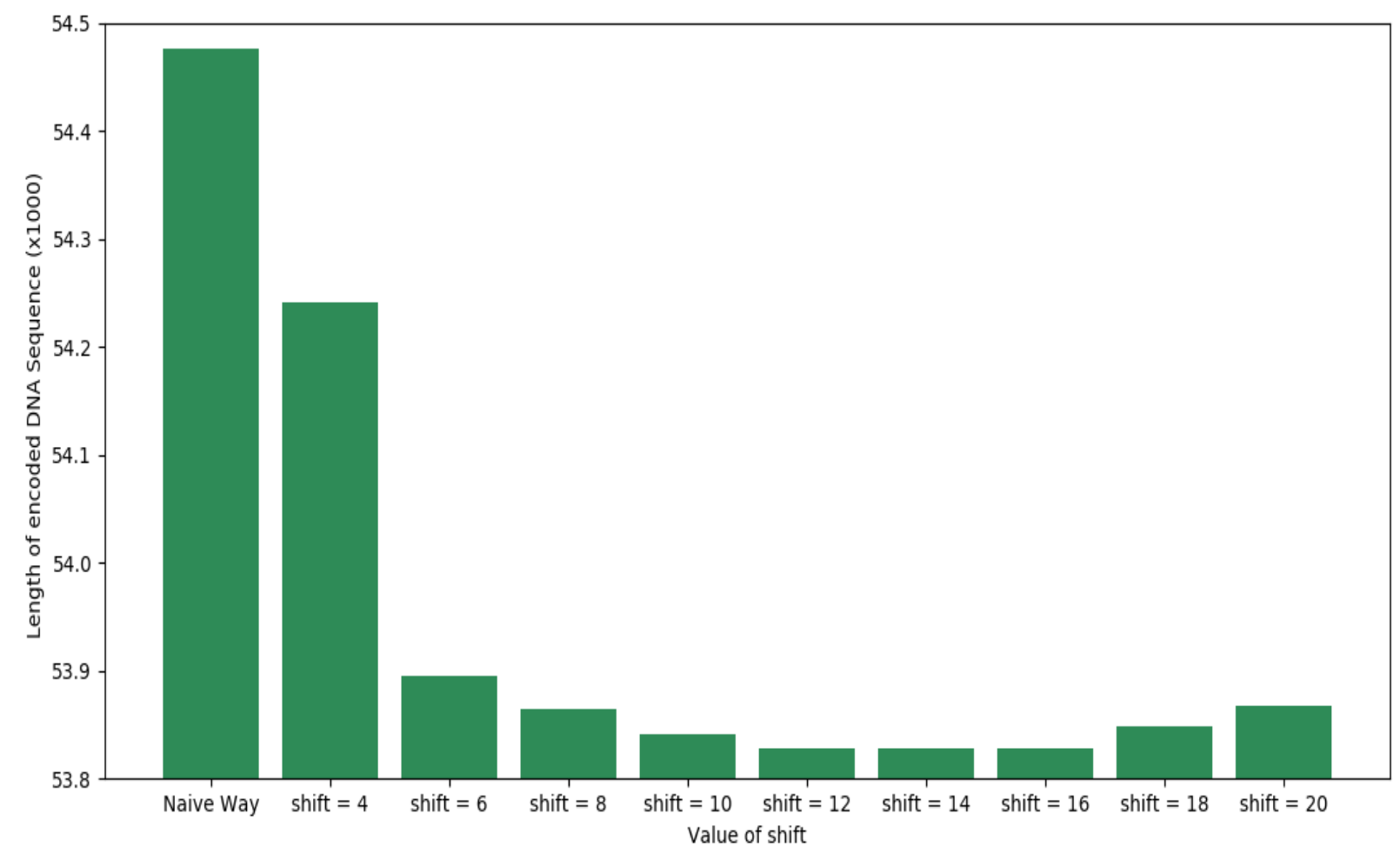

Figure S4: Comparison between our encoding technique (with various shift values) and the naive encoding (without shift parameter). We show the average lengths of the encoded DNA base streams on a single CT scan consisting of 262 DICOM files for the naive encoding and our technique with various values of shift $(4,6,8,10,12,14,16,18$ and 20). The dataset contains 262 DICOM files in a CT scan of the skull collected from: https://wiki.cancerimagingarchive.net/display/Public/APOLLO-1-VA.

\subsection{Compressing encoded DNA sequence}

Once the mapping from binary streams to DNA base streams is done, we further compress the resulting DNA stream to achieve higher storage capacity within the DNA sequence. We performed an extensive evaluation study on various general purpose (Huffman ${ }^{9}$, Deflate ${ }^{4}, \mathrm{RLE}^{3}$, zip, 7zip ${ }^{10}$, bzip2 ${ }^{11}$, gzip ${ }^{12}$, $\mathrm{ANS}^{13}$ etc.) as well as specialized compression techniques (BioCompress- ${ }^{14}, \mathrm{II}^{15}$, DNACompress ${ }^{16}$, DNABit ${ }^{17}$, GenCompress ${ }^{18}$ etc.) for DNA sequence (results not shown). Considering the compression ratio, running time and ease of use of various techniques, we decided to use Deflate in eMED-DNA. 


\subsection{Placement of the dictionary in the genome sequence}

As we have already mentioned, we start storing the dictionary (encoded as DNA stream) from the tail end of the genome. This is opposite to the direction of storing EHR files. If dictionary information were stored alongside the EHR files (from the same end of the genome), dictionary base streams would be interleaved with the EHR files. As dictionary entries produce extremely shorter base sequences than EHR files, the deletion of a file and its corresponding dictionary entry would potentially create small sized free chunks that would be available for storing future EHR files. The EHR files spread over these small free chunks would be fragmented, resulting in more meta information to store, which in turn results into larger dictionary entries. Storing the dictionary from the opposite end of a genome may address these problems.

\section{Free List}

Throughout an entire user session, we maintain a data structure called free list to keep track of the currently available free chunks (i.e., the genome space that are not currently being occupied with EHR files or the dictionary). Each user session starts with initializing the dictionary (by reading the in-DNA dictionary entries from previous user sessions) and constructing the free genome space accordingly.

\subsection{File Operations}

We now briefly describe various file operations within the genome sequence. Each user session of eMED-DNA starts with initializing the dictionary (by reading the in-DNA dictionary entries) and constructing the free list accordingly. eMED-DNA supports three basic file operations: insertion, retrieval and deletion.

\section{Insertion}

New EHR files may be added to a genome. eMED-DNA converts each input file to nucleotide base sequence according to the pipeline described above. For each EHR file, traversing the free list from front to end, the system makes a temporary list of chunks required to accommodate the encoded DNA base stream of that file. Next, a dictionary entry is formed with these locations and other meta data, and converted into DNA base stream. The DNA base stream of the dictionary entry is then inserted into the in-DNA dictionary and the DNA base streams of the EHR files are written into appropriate genomic locations, given that the genome has sufficient free space to accommodate these DNA base streams.

\section{Retrieval}

eMED-DNA can provide a list of the files that have already been stored in the genome sequence by acquiring necessary information from the in-DNA dictionary. eMED-DNA provides a graphical user interface so a user can select a file from this list to view or save a copy to the 
user's computer. Once a file has been selected to view or save, eMED-DNA looks up the corresponding dictionary entry, obtain the genomic locations where this file has been stored and read the encoded DNA base sequences accordingly. Finally, eMED-DNA decodes the DNA base stream into a binary base stream by following a series of decompression and reverse mapping steps.

\section{Deletion}

With well-designed data structures, and EHR and dictionary placement techniques, removing a file from the genome becomes very straight forward. Removing an EHR file may comprise merely removing the corresponding entries in the in-DNA dictionary and updating the free list accordingly. All the entries after the deleted one are shifted by the size of the deleted entry, in order to mitigate fragmentation (similar to the "defragment" operation in an operation system).

\subsection{Additional Results}

Tables S3 and S4 show the distributions of the encoded DNA base streams over various chunks of non-coding regions for some representative EHR files.

Table S3: Distribution of the encoded DNA base stream of a sample DICOM file over various non-coding regions using eMED-DNA. This file requires 70 chunks of non-coding regions of various sizes to fit the 483,610 encoded bases. The sample file is available at https://git.io/fhIDq.

\begin{tabular}{|c|c|c|c|c|c|c|c|}
\hline \multicolumn{8}{|c|}{ File Name: US-PAL-8-10x-echo.dcm (Echocardiogram) } \\
\hline \multicolumn{4}{|c|}{ File Size: 473 KB ( 483610 bytes) } & \multicolumn{4}{|c|}{ Encoded DNA Stream Length: 2104304 bases } \\
\hline \multicolumn{8}{|c|}{ Location in Genome (70 chunks) } \\
\hline $\begin{array}{l}\text { Chunk } \\
\text { No }\end{array}$ & $\begin{array}{l}\text { Chromosome } \\
\text { No }\end{array}$ & $\begin{array}{l}\text { Chunk } \\
\text { Start } \\
\text { Position }\end{array}$ & $\begin{array}{l}\text { Chunk } \\
\text { Size }\end{array}$ & $\begin{array}{l}\text { Chunk } \\
\text { No }\end{array}$ & $\begin{array}{l}\text { Chromosome } \\
\text { No }\end{array}$ & $\begin{array}{l}\text { Chunk Start } \\
\text { Position }\end{array}$ & $\begin{array}{l}\text { Chunk } \\
\text { Size }\end{array}$ \\
\hline 1 & 1 & 0 & 69091 & 36 & 1 & 1540454 & 1219 \\
\hline 2 & 1 & 70009 & 112384 & 37 & 1 & 1574870 & 4886 \\
\hline 3 & 1 & 184159 & 764 & 38 & 1 & 1580047 & 17964 \\
\hline 4 & 1 & 200323 & 250417 & 39 & 1 & 1600097 & 15318 \\
\hline 5 & 1 & 451679 & 234037 & 40 & 1 & 1630611 & 1484 \\
\hline 6 & 1 & 686655 & 238225 & 41 & 1 & 1724325 & 513 \\
\hline
\end{tabular}




\begin{tabular}{|c|c|c|c|c|c|c|c|}
\hline 7 & 1 & 959310 & 1277 & 42 & 1 & 1745993 & 5239 \\
\hline 8 & 1 & 965716 & 781 & 43 & 1 & 1780458 & 4827 \\
\hline 9 & 1 & 982094 & 16868 & 44 & 1 & 1891118 & 23709 \\
\hline 10 & 1 & 1000173 & 965 & 45 & 1 & 1917297 & 293 \\
\hline 11 & 1 & 1014542 & 5581 & 46 & 1 & 1919274 & 2677 \\
\hline 12 & 1 & 1056119 & 14847 & 47 & 1 & 2003838 & 15486 \\
\hline 13 & 1 & 1074308 & 7510 & 48 & 1 & 2030752 & 19718 \\
\hline 14 & 1 & 1116362 & 57522 & 49 & 1 & 2212721 & 15974 \\
\hline 15 & 1 & 1197936 & 5572 & 50 & 1 & 2310120 & 11133 \\
\hline 16 & 1 & 1206692 & 4634 & 51 & 1 & 2391708 & 67 \\
\hline 17 & 1 & 1214139 & 2769 & 52 & 1 & 2413798 & 12182 \\
\hline 18 & 1 & 1232032 & 233 & 53 & 1 & 2505531 & 3002 \\
\hline 19 & 1 & 1235042 & 7404 & 54 & 1 & 2526601 & 2144 \\
\hline 20 & 1 & 1246723 & 7186 & 55 & 1 & 2530246 & 25393 \\
\hline 21 & 1 & 1273886 & 6550 & 56 & 1 & 2565383 & 21108 \\
\hline 22 & 1 & 1292030 & 346 & 57 & 1 & 2632991 & 2985 \\
\hline 23 & 1 & 1324692 & 64 & 58 & 1 & 2801722 & 219761 \\
\hline 24 & 1 & 1328898 & 2416 & 59 & 1 & 3022904 & 46264 \\
\hline 25 & 1 & 1349351 & 3338 & 60 & 1 & 3438622 & 15804 \\
\hline 26 & 1 & 1361778 & 11952 & 61 & 1 & 3481114 & 8806 \\
\hline 27 & 1 & 1375496 & 10215 & 62 & 1 & 3611496 & 13506 \\
\hline 28 & 1 & 1399339 & 2569 & 63 & 1 & 3630128 & 639 \\
\hline 29 & 1 & 1407314 & 11106 & 64 & 1 & 3736202 & 16196 \\
\hline 30 & 1 & 1421770 & 4358 & 65 & 1 & 3771646 & 1142 \\
\hline 31 & 1 & 1427788 & 7073 & 66 & 1 & 3775983 & 2575 \\
\hline
\end{tabular}




\begin{tabular}{|c|c|c|c|c|c|c|c|c|c|}
\hline 32 & 1 & 1442883 & \multicolumn{2}{|c|}{6806} & 67 & \multicolumn{2}{|c|}{1} & 3796505 & 15576 \\
\hline 33 & 1 & 1470159 & \multicolumn{2}{|c|}{1610} & 68 & \multicolumn{2}{|c|}{1} & 3857215 & 52 \\
\hline 34 & 1 & 1497849 & \multicolumn{2}{|c|}{14302} & 69 & \multicolumn{2}{|c|}{1} & 3885430 & 3695 \\
\hline 35 & 1 & 1534688 & \multicolumn{2}{|l|}{486} & 70 & \multicolumn{2}{|c|}{1} & 3900294 & 431709 \\
\hline \multicolumn{10}{|c|}{ in-DNA Dictionary Entry } \\
\hline \multirow{2}{*}{\multicolumn{2}{|c|}{ Length: 105 bases }} & \multirow{2}{*}{\multicolumn{2}{|c|}{$\begin{array}{l}\text { Location in Genome } \\
\text { ( } 1 \text { chunk) }\end{array}$}} & \multicolumn{3}{|c|}{ Chromosome No } & \multicolumn{2}{|c|}{ Chunk Start Position } & $\begin{array}{l}\text { Chunk } \\
\text { Size }\end{array}$ \\
\hline & & & & \multicolumn{3}{|c|}{24} & \multicolumn{2}{|c|}{57227310} & 105 \\
\hline
\end{tabular}

Table S4: Distribution of the encoded DNA base stream of two clinical notes over various non-coding regions using eMED-DNA. Each of these two files requires two chunks (one for the note itself and the other one for the corresponding dictionary entry) of non-coding regions to fit the encoded bases. These notes were obtained from MIMIC-III database ${ }^{19}$.

\begin{tabular}{|c|c|c|c|c|c|c|}
\hline File Name & \multicolumn{3}{|c|}{ NOTEEVENTS-00002.txt } & \multicolumn{3}{|c|}{ NOTEEVENTS-00007.txt } \\
\hline File size & \multicolumn{3}{|c|}{$2.21 \mathrm{~KB}$ (2264 bytes) } & \multicolumn{3}{|c|}{1.77 KB (1813 bytes) } \\
\hline $\begin{array}{l}\text { Encoded DNA } \\
\text { Stream Length }\end{array}$ & \multicolumn{3}{|l|}{5060 bases } & \multicolumn{3}{|l|}{4064 bases } \\
\hline $\begin{array}{l}\text { Dictionary entry } \\
\text { length }\end{array}$ & \multicolumn{3}{|l|}{107 bases } & \multicolumn{3}{|l|}{107 bases } \\
\hline $\begin{array}{l}\text { Location in } \\
\text { genome }\end{array}$ & $\begin{array}{l}\text { Chromosome } \\
\text { No }\end{array}$ & $\begin{array}{l}\text { Chunk } \\
\text { Start } \\
\text { Position }\end{array}$ & $\begin{array}{l}\text { Chunk } \\
\text { Size }\end{array}$ & $\begin{array}{l}\text { Chromosome } \\
\text { no. }\end{array}$ & $\begin{array}{l}\text { Chunk } \\
\text { Start } \\
\text { Position }\end{array}$ & $\begin{array}{l}\text { Chunk } \\
\text { Size }\end{array}$ \\
\hline $\begin{array}{l}\text { EHR file } \\
\text { (1 chunk) }\end{array}$ & 1 & 8111079 & 5060 & 1 & 8116139 & 4064 \\
\hline $\begin{array}{l}\text { Dictionary entry } \\
\text { ( } 1 \text { chunk) }\end{array}$ & 24 & 57227074 & 107 & 24 & 57226967 & 107 \\
\hline
\end{tabular}




\section{Additional Discussion}

This study presents a proof-of-concept for archiving medical records within the human genome sequences. This integration may last as long as thousands of years if preserved under appropriate environment using DNA storage, and thus will contribute towards the advanced research in personalized medicine and future healthcare system. ${ }^{21,22}$ In addition, our study makes significant contribution towards in silico DNA storage technology by introducing several novel techniques in coding theory and operating systems. Our proposed technique for binary-to-DNA conversion is especially tailored for DICOM files but can be used for any digital data.

eMED-DNA provides random access to the records stored in the genome sequence, and may offer the medical practitioners a one-stop solution for easy and efficient storage and management of both genotypes and phenotypes of the patients without having to deal with heterogeneous sources, types and standards of EHR data. eMED-DNA is expected to facilitate the transfer of medical records (along with the genome sequences) across various medical institutions as it can convert and store heterogeneous data into DNA bases and store them as simple computer files.

The ultimate goal of this framework is managing genotype-phenotype information of a patient's lifetime in his DNA sequence. Therefore, we proposed compression techniques especially customized for medical records to accommodate the large amount of EHRs within the limited space available in a genome sequence. However, current compression ratio may not be sufficient to achieve the ultimate goal. Apart from developing advanced compression techniques, we can consider archiving large EHRs in a secured storage platform (for example, cloud storage) and store the link to the remote server along with necessary credentials in the genome sequence so that eMED-DNA can retrieve medical information from remote storage media.

This sort of initiative requires strict policies for privacy issues, and appropriate computational techniques for preventing any possible breach of privacy and information loss to unauthorized parties. Appropriate private key based cryptographic algorithms may be incorporated to eMEDDNA so that in case someone gets a genome sequence with encoded medical records, he will not be able to retrieve the information by using eMED-DNA unless he receives the private key from the original source. In addition to the encryption, strong identity authentication is required which guarantees that the sender and recipient of healthcare data, encoded in genomic sequence, are in fact who they claim to be.

In addition to storing and managing EHRs within ones genome sequence and thus facilitating genomic medicine, it would be interesting to add some other applications (relevant to genomic medicine) in eMED-DNA. For example, genome wide association study (GWAS) considers all the variants in the whole genome to see if any variant is associated to a particular trait or phenotype. The impact of GWAS in medical science could potentially be substantial as it contributes towards understanding the genetic factors contributing to variation in traits and 
diseases. Therefore, options for assisting GWAS by leveraging the genomic data and clinical phenotypes in eMED-DNA will be beneficial.

We believe eMED-DNA will have immediate positive impact in advanced medical research and DNA storage technologies. This will encourage the scientists to develop new architectures or enhance the ones presented in this study for better integration of phenotypic and genomic data. We believe this study will help the scientists to appreciate the need for integrating medical records with genomic data, and drive them towards utilizing this sort of frameworks for precision medicine.

\section{References}

1. Norweck JT, Seibert JA, Andriole KP et al. ACR-AAPM-SIIM technical standard for electronic practice of medical imaging. J Digit Imaging 2013; 26:38-52.

2. Mildenberger P, Eichelberg M, Martin E. Introduction to the DICOM standard. Eur Radiol 2002; 12:920-927.

3. RLE: https://en.wikipedia.org/wiki/Run-length_encoding. (Accessed June 02, 2018.)

4. Deflate: https://en.wikipedia.org/wiki/DEFLATE. (Accessed June 02, 2018.)

5. JPEG : https://jpeg.org/jpeg. (Accessed June 02, 2018.)

6. JPEG LS : https://jpeg.org/jpegls. (Accessed June 02, 2018.)

7. JPEG 2000 : https://jpeg.org/jpeg2000. (Accessed June 02, 2018.)

8. Davis J. Microvenus. Art Journal 1996; 55:70-74.

9. Huffman DA. A Method for the Construction of Minimum-Redundancy Codes. Proceedings of the IRE; 1952; 1098-1101.

10. 7-zip: https://www.7-zip.org/. (Accessed June 02, 2018.)

11. bzip: http://bzip.org/. (Accessed June 02, 2018.)

12. gzip: http://www.gzip.org/. (Accessed June 02, 2018.)

13. Duda J. Asymmetric numeral systems. arXiv preprint 2009; 0902:0271.

14. Grumbach S, Tahi F. Compression of DNA sequences. In: Storer JA, Cohn M, editors. Proceedings of the IEEE Data Compression Conference; 1993 Mar 30-Apr 1; Snowbird, Utah, USA. IEEE Computer Society, 1993:340-350.

15. Grumbach S, Tahi F. A new challenge for compression algorithms: Genetic sequences. Inf Process Manag 1994; 30:875-886.

16. Chen X, Li M, Ma B, Tromp J. DNAcompress: fast and effective DNA sequence compression. Bioinformatics 2002; 18:1696-1698.

17. Rajarajeswari P, Apparao A. DNABIT Compress - Genome compression algorithm. Bioinformation 2011; 5:350-360.

18. Chen X, Kwong S, Li M. A compression algorithm for DNA sequences. IEEE Eng Med Biol Mag 2001; 20:61-66.

19. Johnson AEW, Pollard TJ, Shen L et al. MIMIC-III, a freely accessible critical care database. Scientific Data (2016); 3:160035. 
20. Niedringhaus TP, Milanova D, Kerby MB et al. Landscape of next-generation sequencing technologies. Anal. Chem. 2011; 83:4327-4341.

21. Goldman N, Bertone P, Chen S, et al. Towards practical, high-capacity, low-maintenance information storage in synthesized DNA. Nature 2013;494:77-80.

22. Bornholt J, Lopez R, Carmean DM, Ceze L, Seelig G, Strauss K. A DNA-based archival storage system. Proceedings of the 21th ACM International Conference on Architectural Support for Programming Languages and Operating Systems; 2016 April 2-6; Atlanta, Georgia. ACM New York. NY; 2016. 637-649. 